\title{
Dickkopf Wnt signaling pathway inhibitor 1 regulates the differentiation of mouse embryonic stem cells in vitro and in vivo
}

\author{
LIPING OU, LIAOQIONG FANG, HEJING TANG, HAI QIAO, XIAOMEI ZHANG and ZHIBIAO WANG
}

Key Laboratory of Ultrasound in Medicine and Engineering, College of Biomedical Engineering, Chongqing Medical University, Chongqing 400016, P.R. China

Received January 23, 2015; Accepted September 24, 2015

DOI: $10.3892 / \mathrm{mmr} .2015 .4586$

\begin{abstract}
Embryonic stem cells (ESCs) are pluripotent stem cells derived from early stage embryos. It remains unclear whether inhibiting the $\mathrm{Wnt} / \beta$-catenin signaling pathway using dickkopf Wnt signaling pathway inhibitor 1 (DKK1) impacts on the differentiation potential of mouse ESCs in vitro and in vivo. In the present study, immunohistochemical staining was used to measure the expression of markers of the three germ layers in ESCs and teratomas derived from ESCs. The expression of markers for the Wnt/ $\beta$-catenin signaling pathway were detected by reverse transcription-polymerase chain reaction (RT-qPCR). Immunohistochemistry and western blotting indicated that the expression levels of octamer-binding transcription factor 4 in the DKK1-treated ESC group were significantly greater compared with the control ESCs. Reduced expression levels of NeuroD and bone morphogenetic protein 4 were observed in the DKK1-treated ESCs and teratomas derived from DKK1-treated ESCs compared with the control group. Increased expression levels of SOX17 were observed in the DKK1-treated ESCs compared with the control group. RT-qPCR indicated that $\beta$-catenin expression was significantly reduced in DKK1-treated ESCs and teratomas derived from DKK1-treated ESCs compared with the control groups. Western blotting indicated no alterations in the expression of GSK-3 $\beta$, however, the levels of phosphorylated-GSK-3 $\beta$ were significantly greater in the DKK1 treatment groups, while cyclin D1 and c-Myc expression levels were significantly reduced in the DKK1 treatment groups compared with the control groups. These results suggest that inhibiting Wnt signaling in ESCs using DKK1 may promote mouse ESCs to
\end{abstract}

Correspondence to: Professor Zhibiao Wang or Professor Liaoqiong Fang, Key Laboratory of Ultrasound in Medicine and Engineering, College of Biomedical Engineering, Chongqing Medical University, 1 Yixueyuan Road, Chongqing 400016, P.R. China

E-mail:wangzb@cqmu.edu.cn

E-mail: lqfang06@163.com

Key words: Wnt/ß-catenin signaling, dickkopf-1, embryonic stem cells, differentiation, in vivo differentiate into endoderm in vitro and in vivo, and suppress the tumorigenicity of ESCs.

\section{Introduction}

Embryonic stem cells (ESCs) possess the ability to differentiate into a variety of cell types, and therefore are a source of cells for functional studies and regenerative therapies (1-2). However, the mechanisms of ESC differentiation in vitro and in vivo remain to be fully elucidated. Wnt/ $\beta$-catenin signaling has been demonstrated to promote differentiation, however, not the self-renewal of human embryonic stem cells, and is repressed by octamer-binding transcription factor 4 (OCT4) (3). Wnt proteins are secreted glycoproteins and are regulators of cell proliferation and differentiation, functioning as ligands to stimulate receptor-mediated signal transduction pathways. The activation of the Wnt signaling pathway has been demonstrated to be involved in the pathogenesis of various types of human cancer $(4,5)$. The canonical Wnt (Wnt/ $\beta$-catenin) pathway functions by modulating the translocation of $\beta$-catenin to the nucleus, where it controls critical gene expression programs via interactions with T-cell factor/lymphoid enhancing factor(TCF/LEF) and additional families of transcription factors (6). Under normal conditions, casein kinase $1 \beta(\mathrm{CK} 1 \beta)$ and glycogen synthase kinase-3 $\beta$ $(\mathrm{GSK}-3 \beta)$ sequentially phosphorylate $\beta$-catenin in the Axin complex, which is composed of the scaffolding protein Axin, the tumor suppressor adenomatous polyposis coli gene product, CK1 $\beta$ and GSK-3 $\beta$ (7,8). Phosphorylated $\beta$-catenin is subsequently ubiquitinated, resulting in its proteasomal degradation (9). This process maintains $\beta$-catenin at low levels in the cytoplasm and prevents it from translocating to the nucleus, leading to repression of Wnt target genes (9).

The Wnt pathway is composed of two distinct signaling arms: The canonical and noncanonical pathways. In the canonical pathway, the Wnt protein binds to the cell surface receptor Frizzled, which uses the coreceptors low-density lipoprotein receptor-related protein 5/6 (Lrp5/6) to promote Axin binding to Dishevelled (6). This leads to the stabilization of $\beta$-catenin, which in turn translocates to the nucleus, where it interacts with the DNA-binding proteins from the $\mathrm{TCF} / \mathrm{LEF}$ family to activate transcription of numerous genes (10). Dickkopf Wnt signaling pathway inhibitor 1 (DKK1) is a secreted inhibitor that functions by binding directly to the Lrp5/6 coreceptors 
and inhibiting canonical Wnt signal transduction (11). Previous studies have reported that secreted inhibitors of the Wnt pathway, such as SFRP1 and DKK1, are frequently silenced by methylation in numerous types of human cancer (12).

$\mathrm{Wnt} / \beta$-catenin signaling has been implicated in the maintenance of mouse and human ESCs in vitro (13-20). Additionally, Wnt signaling has been reported to promote the acquisition of pluripotency during the reprogramming of somatic cells to induced pluripotent stem cells $(21,22)$. Numerous studies have demonstrated that activating Wnt/ $\beta$-catenin signaling promotes the self-renewal of mouse ESCs (13-20). Loss-of-function studies have indicated that $\beta$-catenin is required for multilineage differentiation, however, is dispensable for self-renewal $(20,23,24)$. $\beta$-catenin has additionally been demonstrated to exhibit pleiotropic effects in ESCs that are essential for ESC differentiation and the prevention of the acquisition of tumorigenicity (3). These observations demonstrate a role for $\beta$-catenin as a gatekeeper of differentiation and tumorigenesis in ESCs (23).

The role of Wnt/ $\beta$-catenin signaling in ESCs remains unclear, with previous studies reporting contradictory results. In the present study, the Wnt signaling pathway and its regulation by DKK1 was investigated in mouse ESCs. The present study aimed to provide further evidence regarding $\beta$-catenin, and the role of DKK1 on the differentiation potential of ESCs in vitro and in vivo.

\section{Materials and methods}

Cells and animal sources. Five C57BL/6 mice used to establish ESC lines were provided by the Experimental Animal Center of Chongqing Medical University (Chongqing, China). The Balb/c mice (10 male, 10 female; HFK Bioscience Co., Ltd., Beijing, China) were kept under standard conditions and sacrificed at 6-8 weeks old and 18-25 g. This study was approved by the ethics committee of Chongqing Medical University (Chongqing, China).

Extraction of the inner cell mass (ICM) and establishment of ESCs. Uteri were aseptically removed from pregnant mice (3.5 days pregnant), and the blastocyst was flushed with phosphate-buffered saline (PBS). The well-developed blastocysts were collected under a microscope (IX70; Olympus Corporation, Tokyo, Japan). The ICM was digested in trypsin (HyClone Laboratories, Inc., Logan, UT, USA) for 3-5 min. The ICM was gently dispersed using a glass microneedle into small clumps of cells. The ICM was continuously digested into single cells, which were inoculated into a 6-well plate.

In vivo transplantation of ESCs. Mouse ESCs at logarithmic phase were prepared, digested in $0.25 \%$ trypsin, and suspended in PBS. The cell density was adjusted to $1 \times 10^{6}$ cells/100 $\mu \mathrm{l}$. The Balb/c mice were anesthetized and the ESC suspension was injected into the liver of each mouse $(100 \mu \mathrm{l})$. Liver nodules $>0.5 \mathrm{~cm}$ diameter were classified as tumors. Mice were sacrificed by intraperitoneal injection with $1 \%$ sodium pentobarbital $(0.1 \mathrm{ml} / 10 \mathrm{~g}$; Sigma-Aldrich, St. Louis, MO, USA), following disinfection with $0.2 \%$ iodophor (Shandong Lierkang Disinfection Technology Co., Ltd., Shandong, China).
Reagents and antibodies. Rabbit anti-mouse OCT4 (cat. no. ab80892), and rabbit anti-mouse Nanog (cat. no. ab9220) were purchased from Abcam (Cambridge, MA, USA). Rabbit anti-mouse stage-specific embryonic antigen 1 (SSEA1; cat. no. sc-101462) was purchased from Santa Cruz Biotechnology, Inc., Dallas, TX, USA). Goat anti-rabbit IgG fluorescein isothiocyanate (FITC)-conjugated antibodies (cat. no. cw0114) and anti-goat IgG tetramethylrhodamine (TRITC)-conjugated antibodies (cat. no. cw0160) were purchased from Beijing ComWin Biotech Co., Ltd. (Beijing, China). The anti-rabbit streptavidin-peroxidase (SP) immunohistochemical kit (SP-0023) and anti-rat SP immunohistochemical kit (SP-0024) were obtained from BIOSS (Beijing, China). Rabbit anti-mouse NeuroD (cat. no. ab16508) and rabbit anti-mouse bone morphogenic protein 4 (BMP4; cat. no. ab39973) antibodies were from Abcam. Rabbit anti-mouse $\beta$-catenin antibodies (cat. no. 9562) were from Cell Signaling Technology, Inc. (Danvers, MA, USA), anti-GSK3 $\beta$ (cat. no. YT2082) and anti-phosphorylated GSK3 $\beta$ (P-GSK3 $\beta$; cat. no. YP0124) antibodies were from ImmunoWay Biotechnology Company (Newark, DE, USA), and rabbit anti-mouse sex determining region Y-box 17 (SOX17; cat. no. 09-038), anti-c-Myc (cat. no. CBL430-KC) and anti-cyclin D1 (cat. no. ABE52) antibodies were from Merck Millipore (Darmstadt, Germany).

Cell culture and applying exogenous DKK1. C57BL/6 ESC culture medium consisted of: Complete medium $(435 \mathrm{ml}$; Cyagen Biosciences Inc., Guangzhou, China), fetal bovine serum (50 ml; Gibco Life Technologies, Carlsbad, CA, USA), glutamine (5 ml; Gibco Life Technologies), 2-hydrophobic based ethanol $(500 \mu \mathrm{l})$, non-essential amino acids ( $5 \mathrm{ml}$; Gibco Life Technologies), leukemia inhibitory factor (100 $\mu \mathrm{l}$; Gibco Life Technologies) and $10^{5} \mathrm{IU} / 1$ penicillin/100 g streptomycin solution ( $5 \mathrm{ml}$; Gibco Life Technologies). ESCs were added to six-well plates (Thermo Fisher Scientific, Inc., Waltham, MA, USA) containing fetal mouse fibroblasts which had been treated with $10 \mu \mathrm{g} / \mathrm{ml}$ mitomycin C (Roche Diagnostics, Basel, Switzerland) to prevent fibroblast mitosis. Cultures were grown in an incubator containing $5 \% \mathrm{CO}_{2}$ at $37^{\circ} \mathrm{C}$. When cells were adherent and confluent, exogenous recombinant DKK1 (200 ng/ml; PeproTech, Inc., Rocky Hill, NJ, USA) was applied for $24 \mathrm{~h}(19)$.

Immunofluorescent staining. Mouse fibroblast cells treated with $10 \mu \mathrm{g} / \mathrm{ml}$ mitomycin $\mathrm{C}$ were prepared on $8 \mathrm{x} 8 \mathrm{~mm}$ slides with the complete ESC medium replaced at $24 \mathrm{~h}$, and ESCs were subcultured in the fibroblast cell layer at a 1:5 ratio. ESCs were cultured for 48-72 h, following which the original culture medium was discarded. Cells were fixed in $4 \%$ formaldehyde (Beyotime Institute of Biotechnology, Beijing, China) in PBS for $15 \mathrm{~min}$ at room temperature, followed by three washes with $0.1 \%$ bovine serum albumin (BSA; Beyotime Institute of Biotechnology) in PBS. The cells were permeabilized using 0.1\% Triton X-100 (Baisaisi Biotechnology Co., Ltd., Tianjing, China) in PBS containing $0.1 \%$ BSA and $4 \%$ normal goat serum (Gibco Life Technologies) or $10 \%$ donkey serum for SOX17. The cells were incubated with the primary antibodies (anti-SSEA1, anti-OCT4 and anti-Nanog, all at 1:100) overnight at $4^{\circ} \mathrm{C}$, followed by a $1 \mathrm{~h}$ incubation with 
the secondary antibodies (goat anti-rabbit IgG-FITC and anti-goat IgG-TRITC) at room temperature. Subsequent steps were performed according to the manufacturer's instructions for the immunofluorescence kit (BIOSS). Images were acquired using a fluorescence microscope (BX40; Olympus Corporation). Each image contained $>200$ cells and totaled $>2,000$ cells/sample.

Immunohistochemical staining. Feeder layer cells were prepared on $8 \times 8 \mathrm{~mm}$ slides with the complete ESC medium replaced at $24 \mathrm{~h}$, and ESCs were subcultured in the feeder layer at a 1:5 ratio. ESCs were cultured for 48-72 h, following which the original culture medium was discarded. The cells were fixed for $30 \mathrm{~min}$ as above. Following three PBS washes, cells were incubated for $20 \mathrm{~min}$ in $0.5 \%$ Triton X-100. Following three further PBS washes, cells were incubated with $3 \% \mathrm{H}_{2} \mathrm{O}_{2}$ in deionized water for $10-15$ min to eliminate endogenous peroxidase activity. A further three PBS washes were followed by 15 min blocking in normal serum (HyClone Laboratories; GE Healthcare Life Sciences, Logan, UT, USA). The samples were incubated with primary antibodies (anti-SOX17, anti-NeuroD and anti-BMP4, all 1:100) at $4^{\circ} \mathrm{C}$ overnight. Subsequent steps were performed according to the manufacturer's instructions for the anti-rabbit and anti-rat SP immunohistochemical kits. Images were acquired using a confocal microscope (TCS SP8; Leica Microsystems, Wetzlar, Germany). The sections of tumor tissue were fixed and blocked by the same method as for the slides mentioned above. Primary antibodies were used at dilutions of 1:100 and were incubated at $4^{\circ} \mathrm{C}$ overnight. The sections were soaked in an avidin horseradish enzyme tag chain working liquid (ZSGB-BIO Co., Ltd., Beijing, China) at $37^{\circ} \mathrm{C}$ for $15 \mathrm{~min}$ and stained with 3,3'-diaminobenzidine chromogenic substrate and hematoxylin (Wuhan Boster Biological Technology Ltd., Wuhan, China). Images were captured using a microscope (BX40; Olympus Corporation) and were analyzed using Image Pro-Plus version 6.0 (Media Cybernetics, Inc., Rockville, MD, USA). Each image contained $>200$ cells and totaled $>2,000$ cells/sample.

Western blotting for protein expression. Total protein was extracted from the cells using radioimmunoprecipitation assay buffer supplemented with protease (phenylmethylsulfonyl fluoride) and phosphate inhibitors ( $\mathrm{NaF}$ anf $\left.\mathrm{Na}_{3} \mathrm{VO}_{4}\right)$ (Roche Diagnostics) on ice. Protein concentration was determined using a Bicinchoninic Acid protein assay kit (Beyotime Institute of Biotechnology), and loading buffer (5X) was added to the proteins, which were boiled for $5 \mathrm{~min}$. Proteins (50 $\mu \mathrm{g} / \mathrm{lane})$ were fractionated by $12 \%$ SDS-PAGE and electrotransferred onto polyvinylidene difluoride membranes (EMD Millipore, Billerica, MA, USA). The membranes were blocked for $1 \mathrm{~h}$ using $0.1 \%$ Tween-20 (Invitrogen Life Technologies, Carlsbad, CA, USA) and $5 \%$ nonfat milk, prior to incubation with primary antibodies (anti-OCT4, SSEA1, $\beta$-catenin, GSK $3 \beta$, P-GSK3 $\beta$, SOX17, cyclin D1 and c-Myc) at $4^{\circ} \mathrm{C}$ overnight. The membranes were then incubated with horseradish peroxidase-conjugated goat anti-mouse/anti rabbit secondary antibodies (Zhongshan Jinqiao Biotechnology Co., Ltd., Beijing, China) for $1 \mathrm{~h}$ at $37^{\circ} \mathrm{C}$, and antigen antibody complexes were detected by chemiluminescence using the BeyoECL Plus kit (Beyotime Institute of Biotechnology) for 1-3 min. X-ray film (LCNDT
Corporation, Wenzhou, China) was exposed to membranes in a darkroom, fixed and imaged. Optical density (OD) was measured using Quantity One software, version 4.4 (Bio-Rad Laboratories, Inc., Hercules, CA, USA).

Reverse transcription-polymerase chain reaction (RT-PCR). Total RNA from the cells was isolated using TRIzol reagent (Invitrogen Life Technologies) according to the manufacturer's instructions. cDNA was synthesized from 500 ng total RNA using the Takara PCR AMV 3.0 kit (Takara Bio, Inc., Otsu, Japan). PCR was conducted with $1 \mu 1 \mathrm{cDNA}$ in a $20 \mu 1$ reaction volume using a PCR kit(Kangweishiji Biotech Co.,Ltd.,Beijing, China). A negative control was established by using $\mathrm{H}_{2} \mathrm{O}$ as the template. PCR products were detected by $1.5 \%$ agarose gel electrophoresis (BD Biosciences, Franklin Lakes, NJ, USA). The primers used were as follows: GSK-3 $\beta$, forward 5'-TCC CTCAAATTAAGGCACATC-3' and reverse 5'-CACGGT CTCCAGTATTAGCATCT-3'. $\beta$-actin expression served as a control, and the primers were as follows: Forward 5'-GAA GGTGAAGGTCGGAGTC-3' and reverse 5'-GAAGATGGT GATGGGATTTC-3'. PCR was conducted in a thermal cycler (PTC-200; Bio-Rad Laboratories, Inc.) with 35 cycles of denaturation at $94^{\circ} \mathrm{C}$ for $45 \mathrm{sec}$, annealing at $59^{\circ} \mathrm{C}$ for $45 \mathrm{sec}$ and extension at $72^{\circ} \mathrm{C}$ for $45 \mathrm{sec}$ with an initial denaturation of $3 \mathrm{~min}$ and a final extension of $5 \mathrm{~min}$. The amplified products were subjected to gel electrophoresis in $2 \%$ agarose and visualized by ethidium bromide (Sigma-Aldrich) staining under ultraviolet illumination.

Reverse transcription-quantitative PCR (RT-qPCR). Total RNA was extracted from cells using TRIzol reagent according to the manufacturer's instructions and cDNA was generated using a reverse transcription kit (Takara Bio, Inc.) according to the manufacturer's instructions. The PCR primer sequences were as follows: $\beta$-catenin, forward GCTTTCAGTTGAGCT GACCA and reverse AAGTCCAAGATCAGCAGTCTCA; glyceraldehyde 3-phosphate dehydrogenase (GAPDH), forward TGCACCACCAACTGCTTAGC and reverse GGC ATGGACTGTGGTCATGAG. The SYBR Premix ExTaq ${ }^{\mathrm{TM}}$ II kit (Takara Biotechnology Co., Ltd., Dalian, China) was used following the manufacturer's instructions for RT-qPCR, which was performed on an ABI 7500 system (Applied Biosystems Life Technologies, Foster City, CA, USA). PCR cycle parameters were as follows: $94^{\circ} \mathrm{C}$ for $3 \mathrm{~min}$, followed by 30 cycles of $94^{\circ} \mathrm{C}$ for $30 \mathrm{sec}, 56^{\circ} \mathrm{C}$ for $30 \mathrm{sec}$ and $72^{\circ} \mathrm{C}$ for $30 \mathrm{sec}$. The relative expression level of $\beta$-catenin mRNA was calculated using the $2^{-\Delta \Delta C T}$ method. All assays were performed in triplicate.

Statistical analysis. All data were analyzed using one-way analysis of variance using SPSS software, version 10.0 (SPSS, Inc., Chicago, IL, USA). Means were compared using the Student-Newman-Keuls test. $\mathrm{P}<0.05$ was considered to indicate a statistically significant difference.

\section{Results}

The growth state of ESCs in the DKK1-treated and control groups. Isolated murine ESCs in the control group exhibited morphological features characteristic of ESCs: Active proliferation, closely connected cells, "nest" growth, clear colony 
A

a

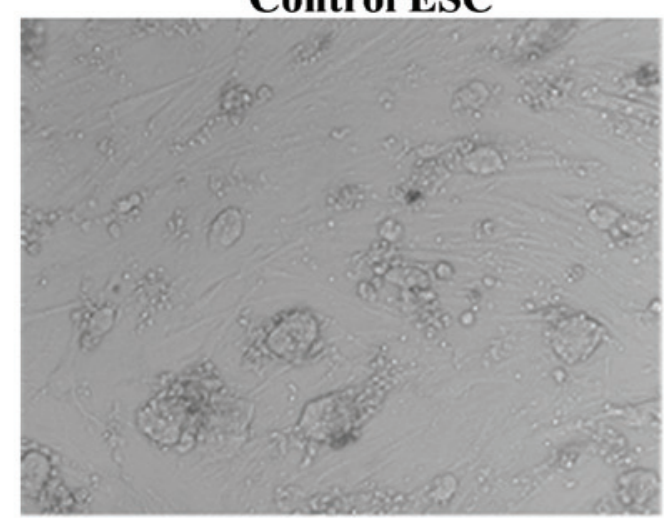

c

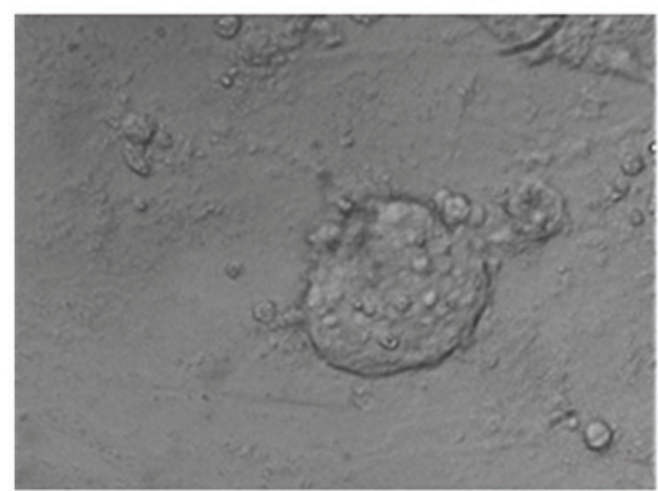

B

Control ESC

Control ESC

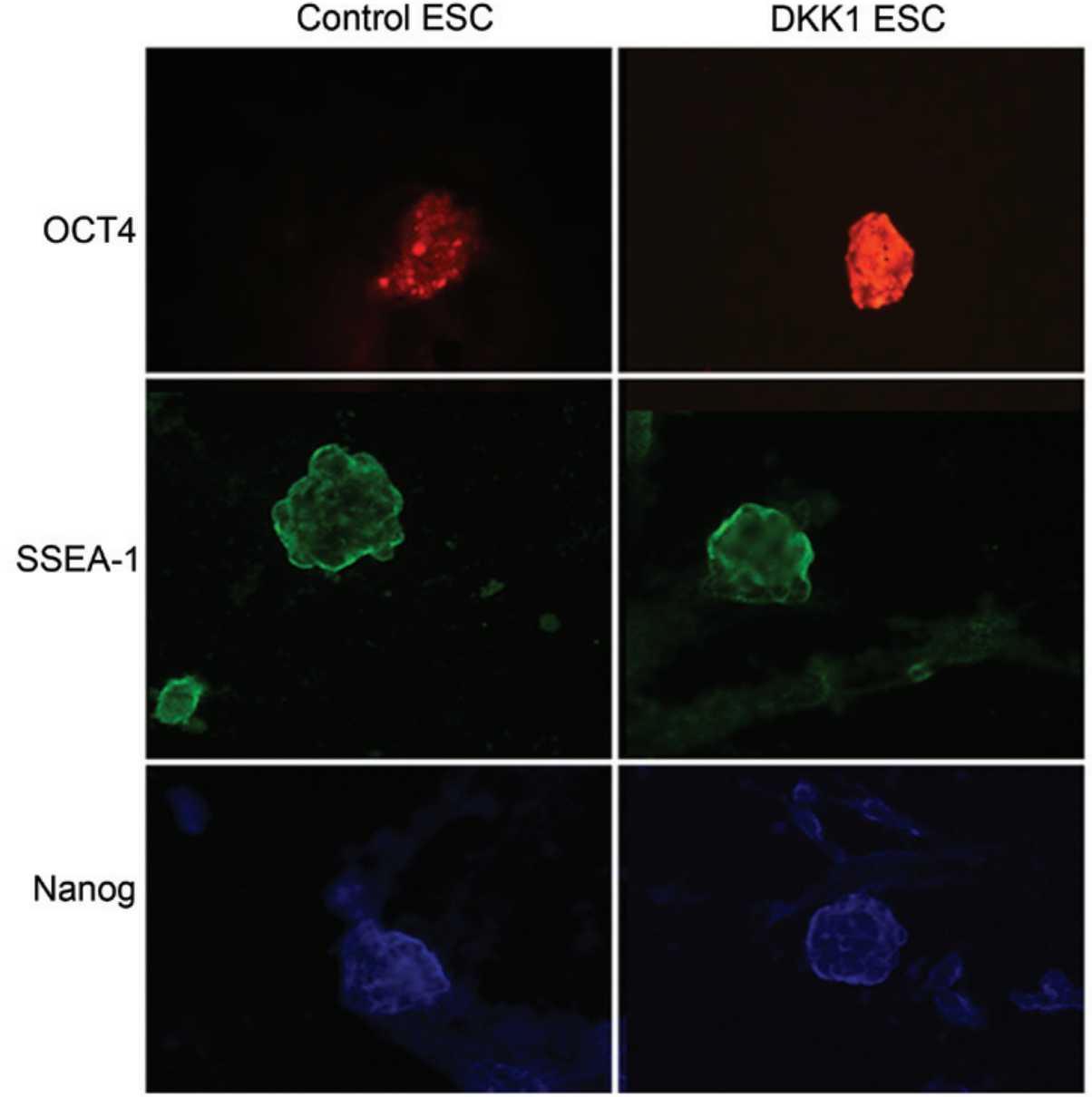

DKK1 ESC
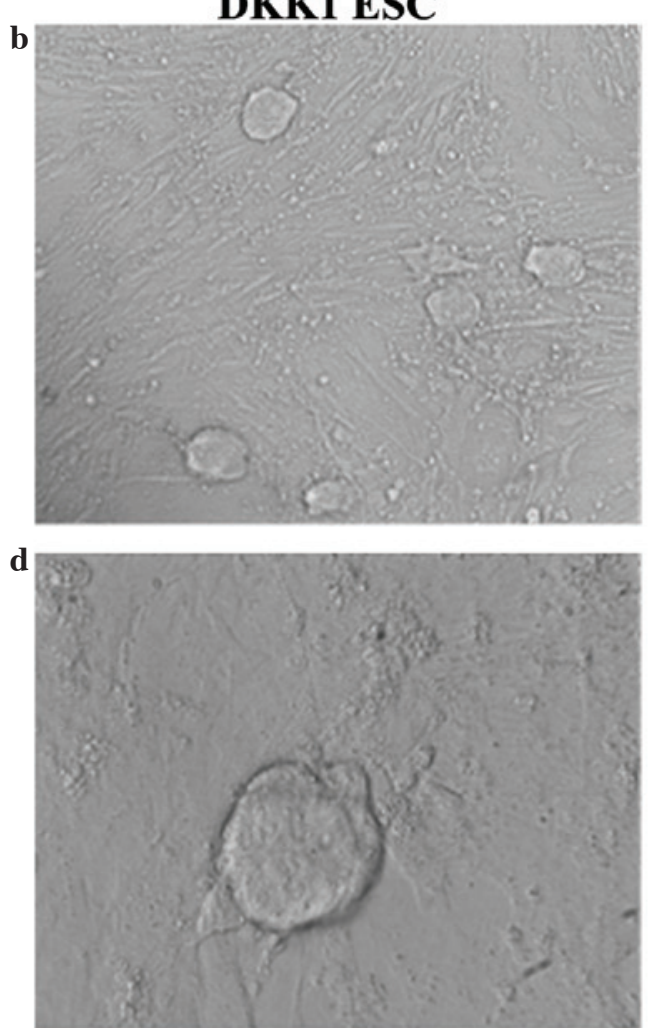

DKK1 ESC 
C
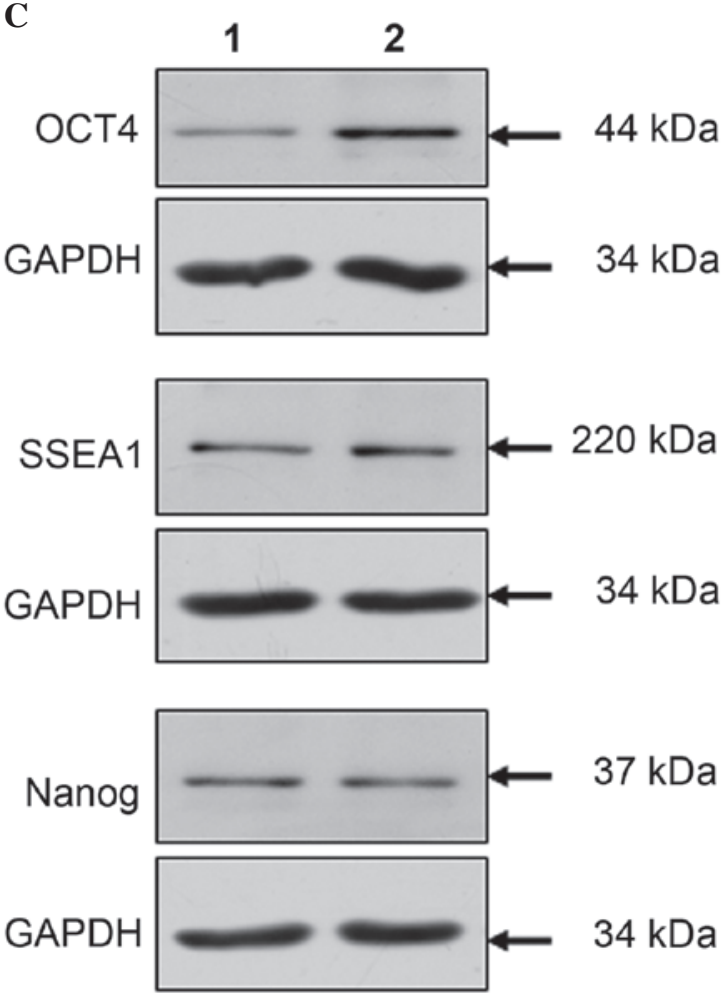

$44 \mathrm{kDa}$

$34 \mathrm{kDa}$

$34 \mathrm{kDa}$

$34 \mathrm{kDa}$
D

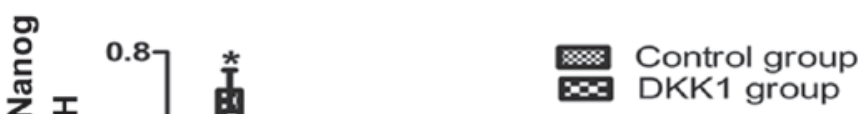

Figure 1. Continued. (C) Western blot analysis of OCT4, SSEA1 and Nanog expression in control ESCs and DKK1-treated ESCs: Lane 1, control ESC group; lane 2, DKK1-treated ESCs. (D) Histogram for western blot analysis results for OCT4, SSEA1 and Nanog in control ESCs and DKK1-treated ESCs. "P<0.05 vs. control. The abundance of GAPDH was determined as a control. Values are presented as the mean \pm standard deviation ( $\mathrm{n}=3$ ). OCT4, octamer-binding transcription factor 4; SSEA1, stage-specific embryonic antigen 1; ESC, embryonic stem cell; DKK1, dickkopf Wnt signaling pathway inhibitor 1; GAPDH, glyceraldehyde 3-phosphate dehydrogenase.
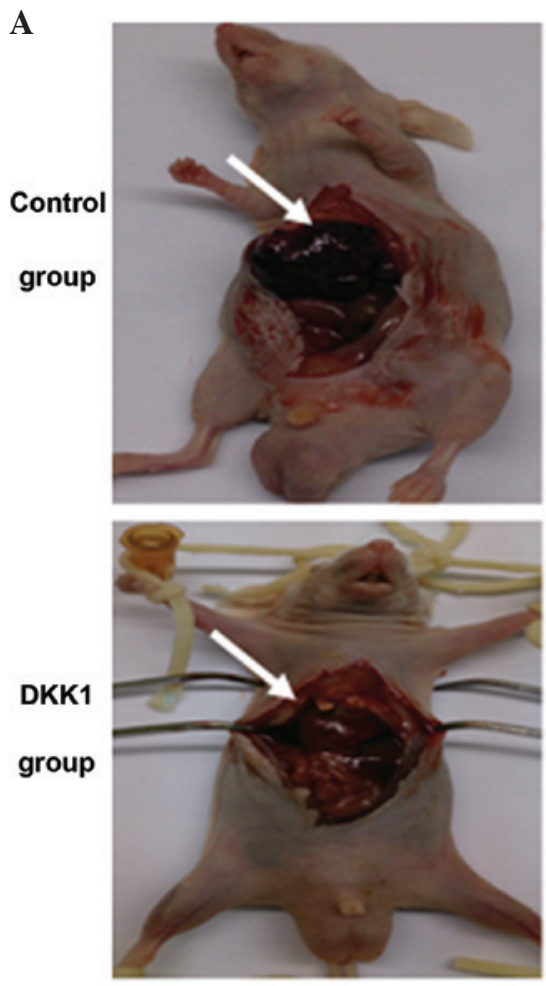
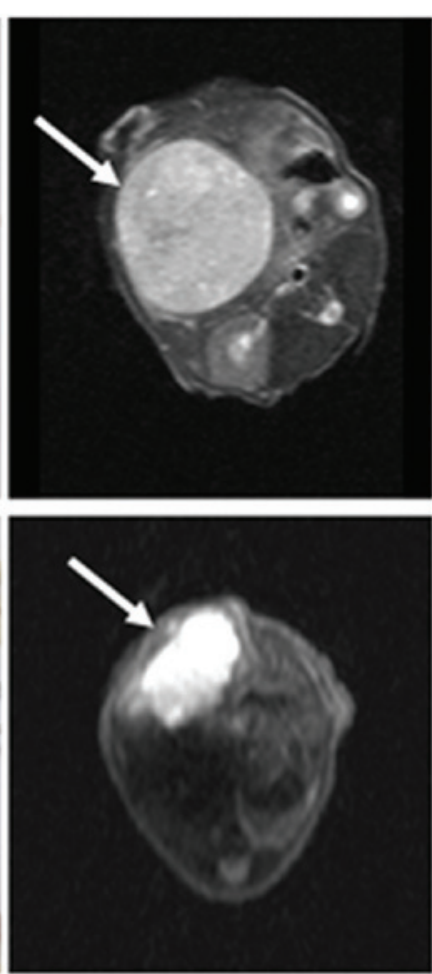
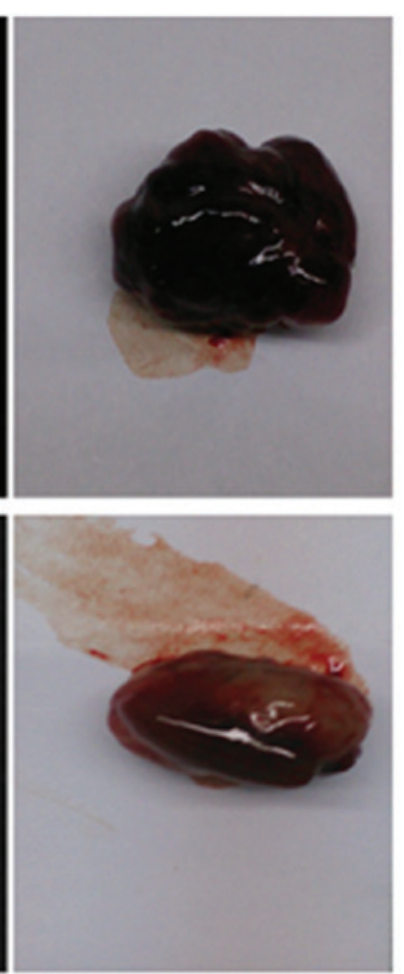

B

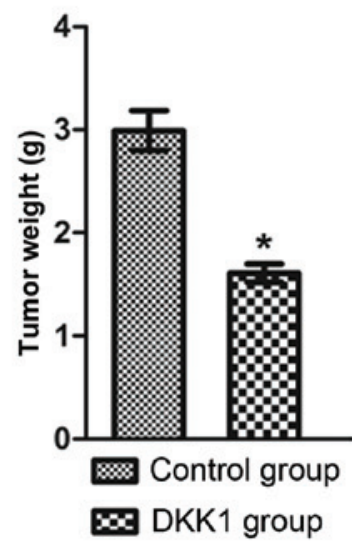

Figure 2. The liver teratomas and the teratoma tissue sections were stained by hematoxylin and eosin following transplantation with control ESCs and DKK1-treated ESCs. (A) Liver teratoma derived from control ESCs 4 weeks post transplantation. The middle column is a magnetic resonance imaging scan. (B) Histogram of the tumor weight of liver teratomas. Data are presented as the mean \pm standard deviation. " $\mathrm{P}<0.05$ vs. control. ESC, embryonic stem cell; DKK1, dickkopf Wnt signaling pathway inhibitor 1 . 
C

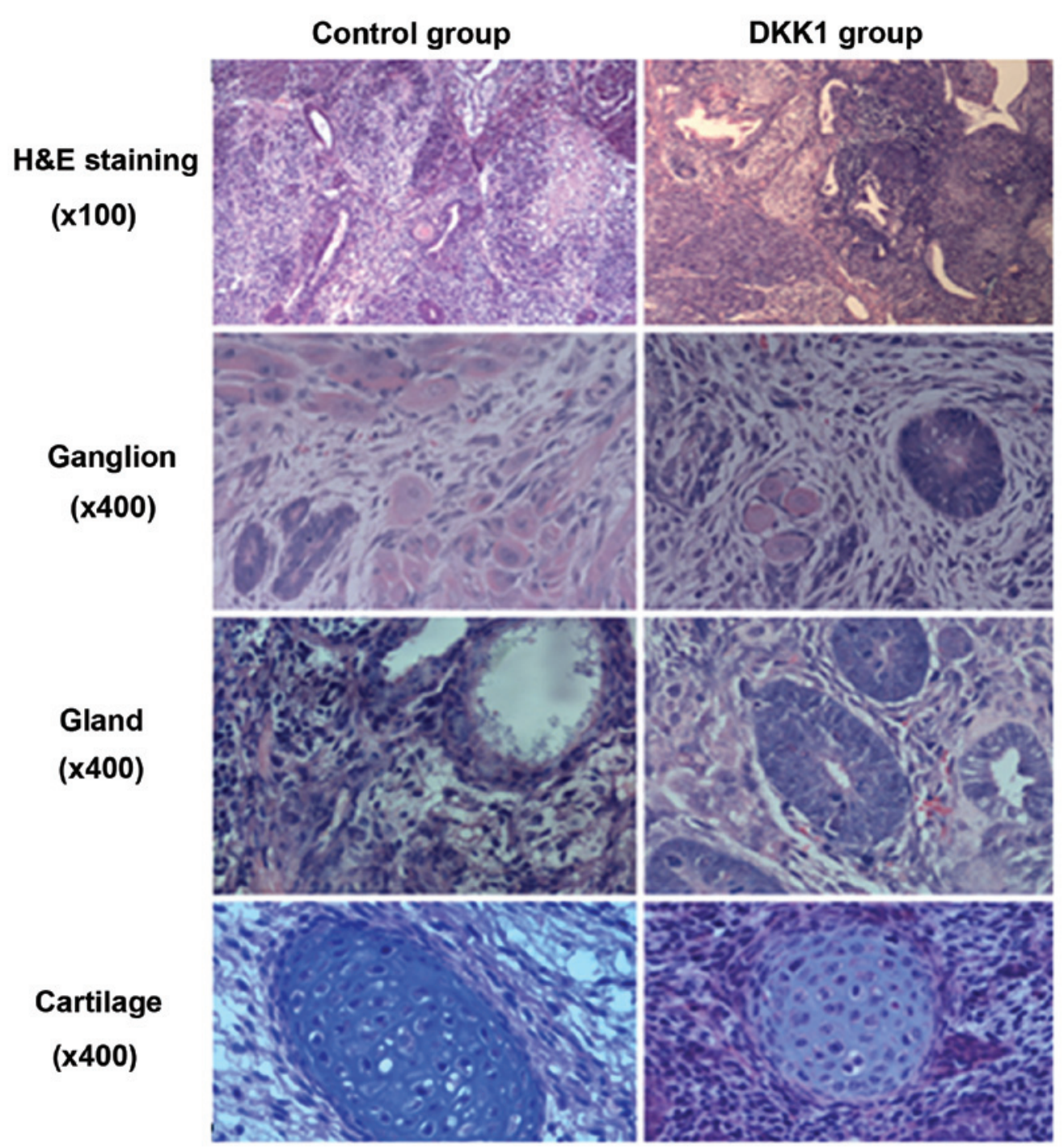

Figure 2. Continued. (C) Hematoxylin and eosin staining for teratomas derived from control ESCs and DKK1-treated ESCs (magnification, x100). Ganglion, glandular and cartilage tissues in teratomas derived from control and DKK1-treated ESCs are presented (magnification, x400). ESC, embryonic stem cell; DKK1, dickkopf Wnt signaling pathway inhibitor 1.

edges with smooth surfaces, dense structure and clear boundaries between the layers of cells (Fig. 1A). The colony of ESCs in the DKK1-treated group appeared to be clearer and more rounded compared with those in the control group (Fig. 1A).

OCT4, SSEAl and Nanog expression in ESCS. Immunofluorescent staining was conducted for OCT4, SSEA1 and Nanog, which indicated postive expression in the control ESCs for OCT4, SSEA1 and Nanog (Fig. 1B). In the DKK1-treated ESC group, the expression of OCT4 appeared greater compared with the control group, however, the expression levels of SSEA1 and Nanog were comparable to the levels observed in the control group.

Western blot analysis of OCT4, SSEA1 and Nanog expression in ESCs. The protein expression levels of OCT4, SSEA1 and Nanog in ESCs were analyzed by western blotting and were quantified (Fig. 1C and D). This indicated positive expression of OCT4 protein in the control ESCs, however, significantly greater expression of OCT4 was observed in DKK1-treated
ESCs $(\mathrm{P}<0.05$; Fig 1D). The expression levels of SSEA1 in control ESCs were not significantly different compared with the DKK1-treated ESCs. In addition, the expression levels of Nanog in control ESCs were not significantly different compared with the DKK1-treated ESCs.

Histological pathology of liver teratomas. The teratomas in the control group and the DKK1-treated group were collected 4 weeks following transplantation, and the tumor weight was measured. The weight of the tumors from the DKK1-treated group was significantly reduced compared with the control group (Fig. 2A and B). Sections of tumor tissue from each group were stained with hematoxylin and eosin to investigate the presence of the three germ layers (Fig. 2C). In the control ESC group, the liver teratomas exhibited primarily glandular epithelial structure, with an atypical cell and tissue structure, disordered cell size, hyperchromatic nuclei, increased nuclear fission, irregular mitosis and sections of necrosis. Histopathological evaluation indicated that the lesion area had formed a mature teratoma of epithelial cancer (Fig. 2C). 


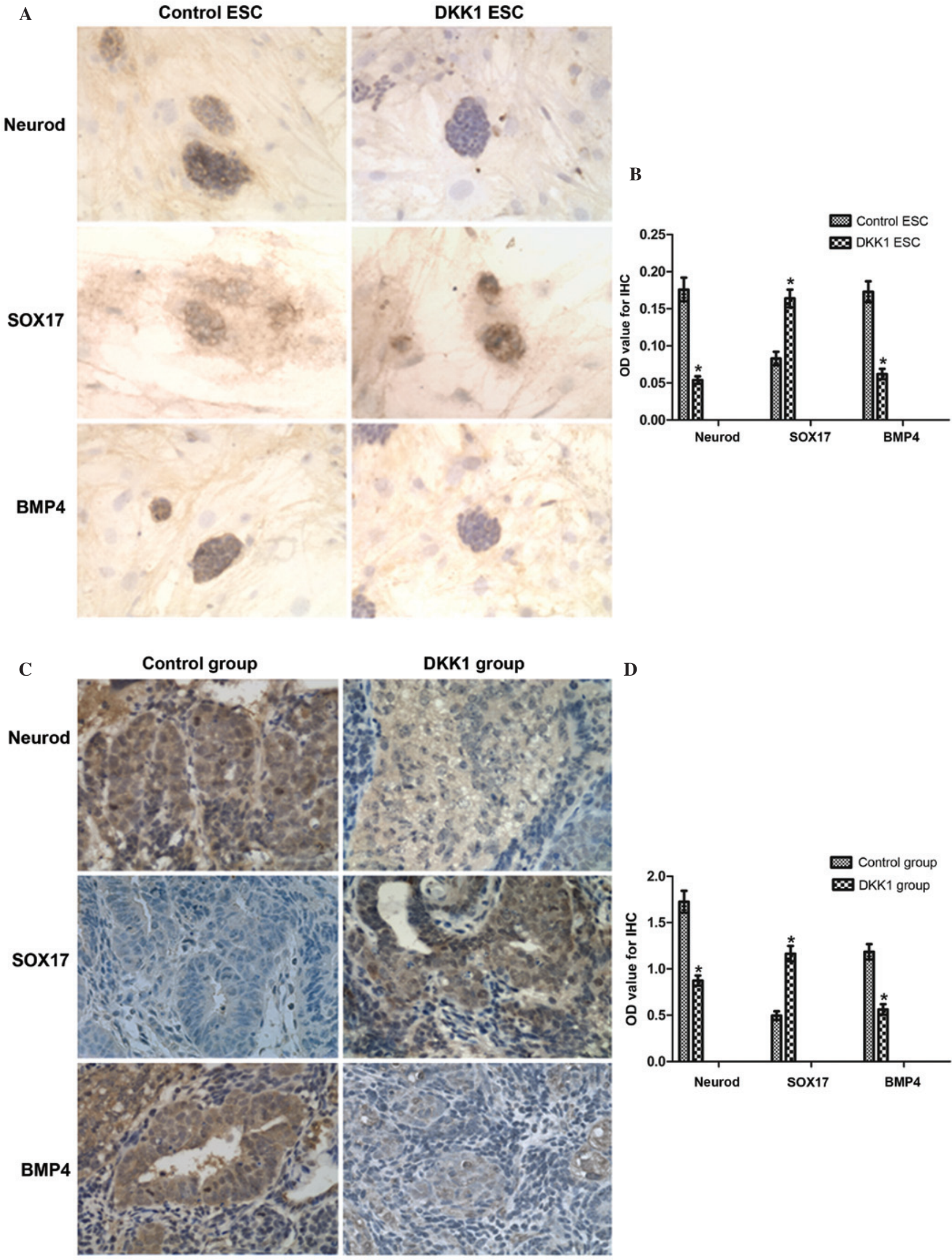

Figure 3. IHC staining for NeuroD, SOX17 and BMP4 expression in ESCs and derived teratomas. (A) NeuroD, SOX17 and BMP4 expression in ESC groups by IHC staining (magnification, x200). (B) Quantification of IHC staining for NeuroD, SOX17 and BMP4. (C) NeuroD, SOX17 and BMP4 expression in teratomas derived from control ESCs and DKK1-treated ESCs (magnification, x400). (D) Quantification of IHC staining for NeuroD, SOX17 and BMP4. "P<0.05 vs. control. IHC, immunohistochemical; SOX17, sex determining region Y-box 17; BMP4, bone morphogenic factor 4; ESC, embryonic stem cell; DKK1, dickkopf Wnt signaling pathway inhibitor 1; OD, optical density. 
A

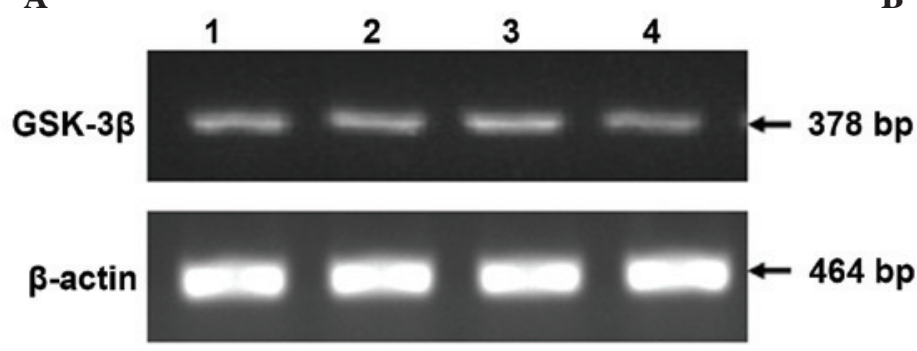

$\mathbf{C}$

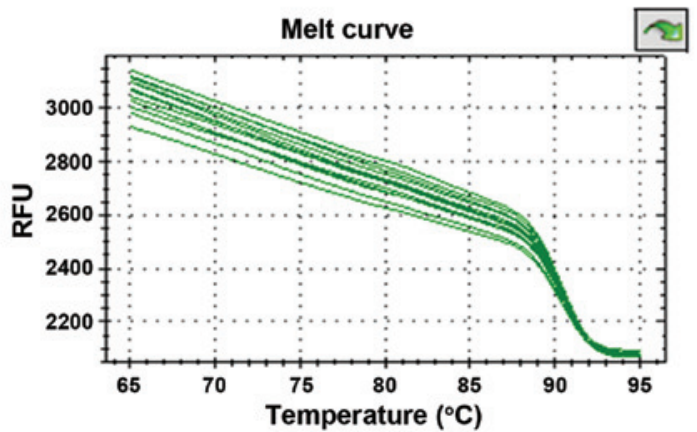

B

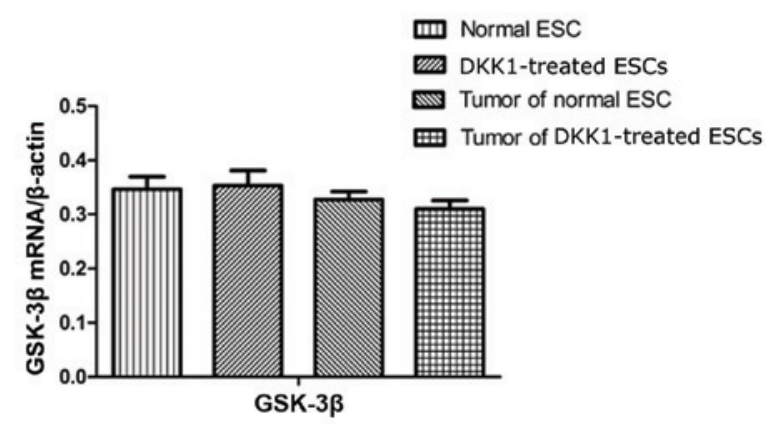

D

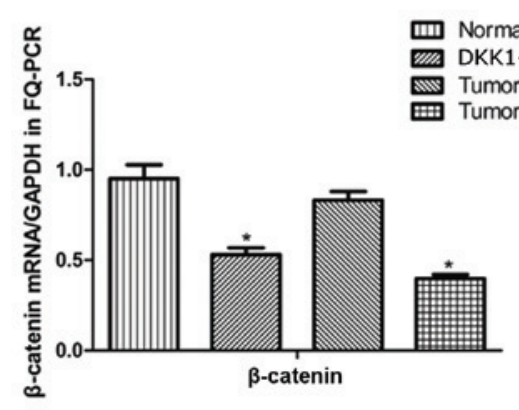

Figure 4. RT-PCR analysis for GSK-3 $\beta$ mRNA and RT-qPCR for $\beta$-catenin mRNA. (A) Following 35 cycles, $5 \mu 1$ samples were separated electrophoretically through the agarose gel, and the expected product at 378 base pairs for GSK-3 $\beta$ were stained with ethidium bromide. Lane 1, control ESC; lane 2, DKK1-treated ESCs; lane 3, teratoma tissue derived from control ESCs; lane 4, teratoma tissue derived from DKK1-treated ESCs. (B) Histogram of GSK-3 $\beta$ mRNA expression by RT-PCR. (C) Melt curve of RT-qPCR for $\beta$-catenin. (D) Histogram for $\beta$-catenin by RT-qPCR. ${ }^{*} \mathrm{P}<0.05$ vs. control. The abundance of $\beta$-actin or GAPDH was used as a control. Values are presented as the mean \pm standard deviation $(n=3)$. RT-PCR, reverse transcription-polymerase chain reac-

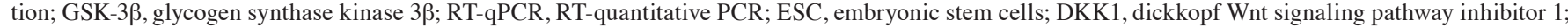
GAPDH, glyceraldehyde 3-phosphate dehydrogenase; RFU, relative fluorescence units.

In the DKK1-treated ESC group, the liver teratomas had a primarily glandular epithelial structure, with atypical cell and tissue structure. However, the ganglia were reduced in number compared with the control group, and the cartilage tissue was observed to be less mature compared with the control group. Furthermore, tissue from the digestive gland demonstrated increased numbers of cells compared with the control group, with a clearer structure than that of the control group (Fig. 2C).

NeuroD, SOX17 and BMP4 expression in ESCs. Staining for the markers of the three germ layers indicated that DKK1-treatment resulted in altered expression in ESCs compared with the control (Fig. 3A and B). Expression of NeuroD was reduced in the DKK1-treated group compared with the control group $(\mathrm{P}<0.05$; Fig. $3 \mathrm{~B})$. SOX17 staining demonstrated increased expression in the DKK1-treated ESCs compared with the control group ( $\mathrm{P}<0.05$; Fig. 3B). Expression of BMP4 was reduced in the DKK1-treated ESCs compared with the control group $(\mathrm{P}<0.05$; Fig. $3 \mathrm{~B})$.

NeuroD , BMP4 and SOX17 expression in the teratomas. As presented above, the markers of the three germ layers additionally exhibited alterations in expression between the teratomas derived from the control and DKK1-treated ESCs (Fig. 3C and D). Staining for NeuroD expression in tumors derived from control ESCs is presented in Fig. 3C, and this indicated that in the DKK1-treated group, NeuroD exhibited reduced expression compared with the control group $(\mathrm{P}<0.05$; Fig. 3D). Staining for BMP4 expression indicated reduced expression in tumors from the DKK1-treated group compared with the control group ( $\mathrm{P}<0.05$; Fig. 3D). However, staining for SOX17 expression indicated increased expression in the DKK1-treated group compared with the control group $(\mathrm{P}<0.05$; Fig. 3D).

RT-PCR analysis for GSK-3 $\beta$ mRNA expression in ESCs and teratoma tissues. Expression levels of GSK-3 $\beta$ mRNA were analyzed by RT-PCR. This indicated that there was no significant difference in the expression of GSK-3 $\beta$ mRNA between the control ESCs, DKK1-treated ESCs, control teratomas or DKK1-treated teratomas ( $\mathrm{P}>0.05$; Fig. 4B).

$R T$ - $q P C R$ analysis of $\beta$-catenin mRNA in ESCs and teratomas. To gain further insight into the molecular mechanisms underlying the differential expression of the markers, the mRNA levels of $\beta$-catenin were measured in ESCs and the derived teratomas. RT-qPCR was used to examine the mRNA expression of $\beta$-catenin, which is associated with the Wnt signaling pathway. This demonstrated that $\beta$-catenin expression was significantly reduced in DKK1-treated ESCs and teratomas derived from DKK1-treated ESCs when compared with the control group $(\mathrm{P}<0.05$; Fig. $4 \mathrm{C}$ and $\mathrm{D})$.

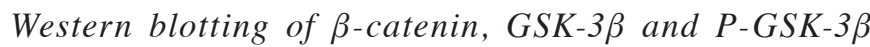
expression in ESCs and teratomas. The expression levels of key proteins in the Wnt signaling pathway were measured in ESCs and derived tumor tissues using western blotting. This indicated clear expression of $\beta$-catenin in control ESCs and teratomas derived from control ESCs whilst expression was significantly reduced in DKK1-treated ESCs and 

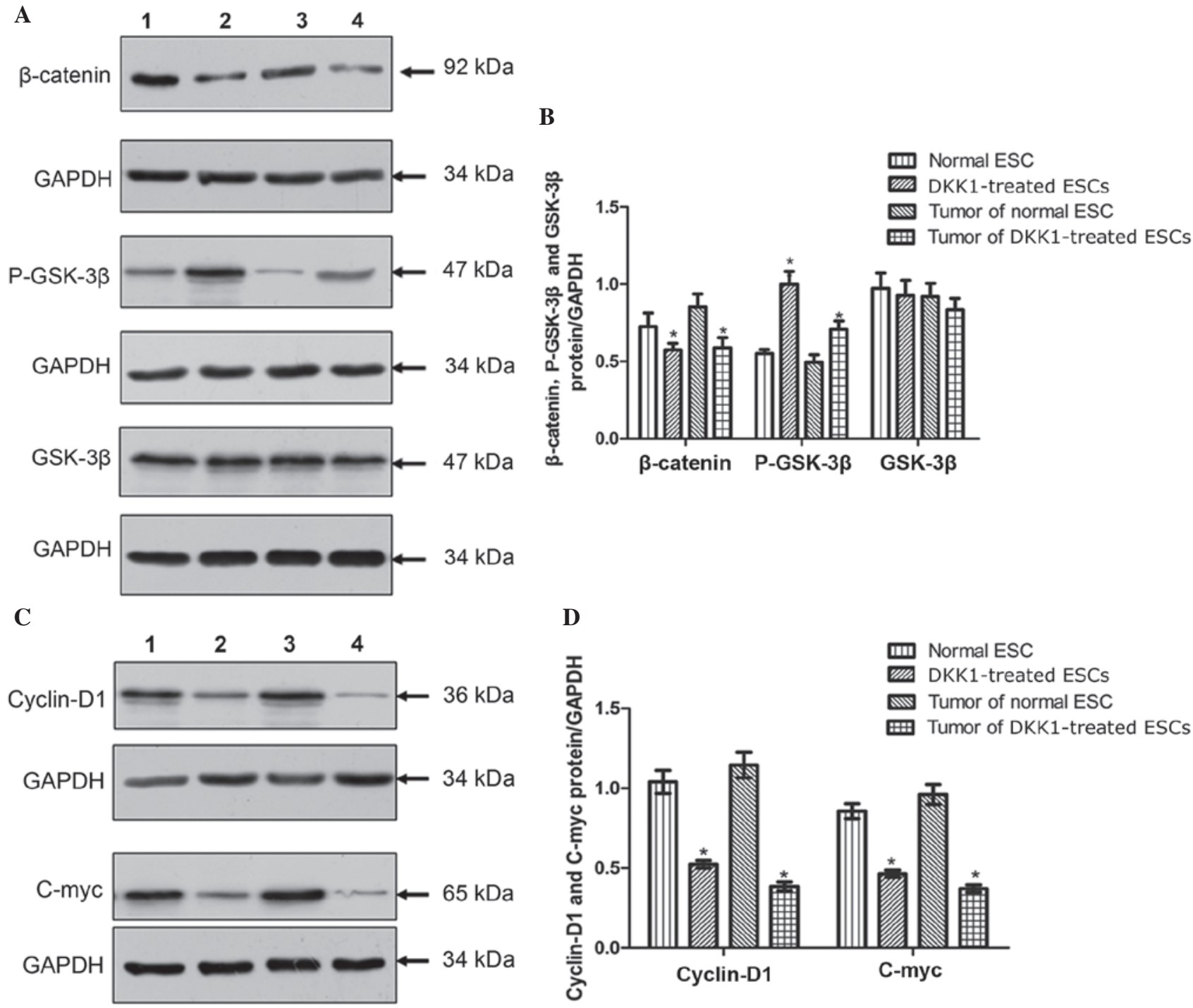

Figure 5. Western blot analysis for the expression of molecules in the Wnt/ $\beta$-catenin signaling pathway. (A) Western blotting images of $\beta$-catenin, P-GSK-3 $\beta$ and GSK-3 $\beta$ : Lane 1, control ESCs; lane 2, DKK1-treated ESCs; lane 3, teratoma derived from control ESCs, lane 4, teratoma derived from DKK1-treated ESCs. (B) Quantification of western blotting for $\beta$-catenin, P-GSK-3 $\beta$ and GSK-3 $\beta$. (C) Western blotting images of cyclin D1 and c-Myc. (D) Quantification of western blotting for cyclin D1 and c-Myc. "P<0.05 vs. control. GAPDH was used as a control. Values are presented as the mean \pm standard deviation (n=3).

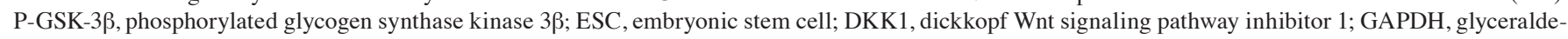
hyde 3-phosphate dehydrogenase.

teratomas derived from DKK1-treated ESCs compared with controls (Fig. 5A and B). The expression of P-GSK-3 $\beta$ was significantly increased in DKK1-treated ESCs and derived teratomas compared with control ESCs and teratomas $(\mathrm{P}<0.05$, Fig. 5A and B). Whilst the expression levels of P-GSK-3 $\beta$ were altered by DKK1 treatment, the expression levels of GSK- $3 \beta$ were not significantly different between the groups $(\mathrm{P}>0.05$, Fig. 5A and B).

Western-blotting for cyclin D1 and c-Myc expression. The expression levels of additional key proteins in the Wnt signaling pathway were measured in ESCs and tumor tissues. This demonstrated that the expression levels of cyclin D1 were reduced in DKK1-treated ESCs and tumors derived from DKK1-treated ESCs compared with control ESCs and the corresponding tumors $(\mathrm{P}<0.05$; Fig. $5 \mathrm{C}$ and $\mathrm{D})$. In addition, the expression levels of c-Myc in DKK1-treated ESCs and tumors derived from DKK1-treated ESCs were significantly reduced compared with the expression in control ESCs and tumors derived from control ESCs ( $\mathrm{P}<0.05$; Fig. $5 \mathrm{C}$ and $\mathrm{D})$.

\section{Discussion}

In the current study, the staining of typical ESC antigens was used to characterize ESCs. The expression of OCT4, SSEA1 and Nanog was observed, verifying the identity of the cells as ESCs. It was observed that the treatment with DKK1 resulted in improved cellular morphology and an increase in OCT4 expression. Variation in the inhibitory effects of Wnt/ $\beta$-catenin signaling in ESCs has been observed in previous studies, and a potential explanation for this may involve Wnt repression. A previous study supported a model whereby Wnt/ $\beta$-catenin signaling is repressed by OCT4 in the context of self-renewing human ESCs (hESCs) and is derepressed when hESCs differentiate (3). In the current study, liver teratomas derived from control ESCs in vivo, HE staining indicated that tumor tissue 
consisted predominantly of glandular epithelial structures that formed a mature teratoma. Neoplastic cells assumed a nest-like morphology and pathological tests confirmed epithelioid cancerous progression. The current study identified differential alterations in the markers of the three germ layers when DKK1-treated ESCs were injected into mice. The NeuroD staining of the ectoderm and the BMP4 staining of the mesoderm exhibited reduced expression in the DKK1-treated ESC groups in vitro and in vivo, whilst SOX17 staining of the endoderm indicated increased expression compared with the control group. These results suggest that the inhibition of Wnt/ $\beta$-catenin signaling may have promoted endoderm differentiation.

In addition, Wnt signaling has been reported to promote pluripotency during the reprogramming of somatic cells to induce pluripotent stem cells $(25,26)$. However, studies have reported that inhibiting Wnt3A or GSK3 results in the differentiation of hESCs towards the primitive streak and definitive endoderm lineages $(27,28)$. Davidson et al (3) reported a primary role for $\mathrm{Wnt} / \beta$-catenin signaling in the differentiation, rather than the self-renewal, of hESCs in vitro. This was demonstrated through sustained inhibition of the pathway, which did not prevent the expansion of hESCs, suggesting that endogenous $\mathrm{Wnt} / \beta$-catenin signaling is not required for the self-renewal of undifferentiated hESCs. Furthermore, the activation of $\beta$-catenin signaling resulted in an induction of mesoderm lineage transcripts and an eventual loss of self-renewal following several passages (3). In the current study, Wnt/ $\beta$-catenin signaling was not required for the self-renewal of undifferentiated ESCs however, was required for the differentiation of ESCs. The expression levels of $\beta$-catenin were significantly reduced in DKK1-treated ESCs and teratomas derived from DKK1-treated ESCs compared with the control groups. It was demonstrated that P-GSK-3 $\beta$ expression was significantly greater in the DKK1 treatment groups, with no effect on total GSK-3 $\beta$ levels. The expression levels of cyclin D1 and c-Myc were significantly reduced in the DKK1 treatment groups compared with the control groups. These results indicate that the $\mathrm{Wnt} / \beta$-catenin signaling pathway regulated the differentiation of ESCs through OCT4 and $\beta$-catenin. Cyclin D1 serves a critical role in the regulation of cell cycle progression, and a TCF4 binding site is located in its promoter region, suggesting the involvement of the $\beta$-catenin/TCF4 pathway in the regulation of cyclin D1 expression (29). The current study additionally indicated that the levels of cyclin D1 and c-Myc protein expression were significantly reduced in teratomas derived from DKK1-treated ESCs compared with teratomas derived from control ESCs. The tumor weight in the DKK1-treated ESC group was significantly reduced compared with the control ESC group. This indicated that the $\mathrm{Wnt} / \beta$-catenin signaling pathway may additionally be associated with the proliferation and tumorigenicity of ESCs.

In conclusion, the current study demonstrated that the Wnt signaling pathways were regulated by the inhibitor DKK1 in ESCs. DKK1 was demonstrated to antagonize the Wnt $/ \beta$-catenin pathway via a reduction in $\beta$-catenin and an increase in OCT4 expression. In addition, the present study suggests that the Wnt/ $\beta$-catenin signaling pathway was associated with the direction of differentiation of ESCs in vitro and in vivo. Furthermore, the current study identified a novel molecular interaction between DKK1 and the Wnt/ $\beta$-catenin pathway during ESC development.

\section{Acknowledgements}

The current study was supported by a project grant from the National Basic Research Program of China (973 program; grant no. 2011CB707900).

\section{References}

1. Doetschman TC, Eistetter H, Katz M, Schmidt W and Kemler R: The in vitro development of blastocyst-derived embryonic stem cell lines: Formation of visceral yolk sac, blood islands and myocardium. J Embryol Exp Morphol 87: 27-45, 1985.

2. Keller G: Embryonic stem cell differentiation: Emergence of a new era in biology and medicine. Genes Dev 19: 1129-1155, 2005.

3. Davidson KC, Adams AM, Goodson JM, McDonald CE, Potter JC, Berndt JD, Biechele TL, Taylor RJ and Moon RT: Wnt/ $\beta$-catenin signaling promotes differentiation, not self-renewal, of human embryonic stem cells and is repressed by Oct4. Proc Natl Acad Sci USA 109: 4485-4490, 2012.

4. Karim R, Tse G, Putti T, Scolyer R and Lee S: The significance of the Wnt pathway in the pathology of human cancers. Pathology 36: 120-128, 2004.

5. Moon RT, Kohn AD, De Ferrari GV and Kaykas A: WNT and $\beta$-catenin signalling: Diseases and therapies. Nat Rev Genet 5: 691-701, 2004.

6. Daniels DL and Weis WI: $\beta$-catenin directly displaces Groucho/TLE repressors from Tcf/Lef in Wnt-mediated transcription activation. Nat Struct Mol Biol 12: 364-371, 2005.

7. Huang $\mathrm{H}$ and $\mathrm{He} X$ : Wnt/ $\beta$-catenin signaling: New (and old) players and new insights. Curr Opin Cell Biol 20: 119-125, 2008.

8. MacDonald BT, Tamai $\mathrm{K}$ and $\mathrm{He} \mathrm{X}$ : Wnt/ $\beta$-catenin signaling: Components, mechanisms, and diseases. Dev Cell 17: 9-26, 2009.

9. van Amerongen R and Nusse R: Towards an integrated view of Wnt signaling in development. Development 136: 3205-3214, 2009.

10. Gadue P, Huber TL, Paddison PJ and Keller GM: Wnt and TGF-beta signaling are required for the induction of an in vitro model of primitive streak formation using embryonic stem cells. Proc Natl Acad Sci USA 103: 16806-16811, 2006.

11. Nakamura T, Sano M, Songyang Z and Schneider MD: A Wntand beta -catenin-dependent pathway for mammalian cardiac myogenesis. Proc Natl Acad Sci USA 100: 5834-5839, 2003.

12. Monzen K, Shiojima I, Hiroi Y, Kudoh S, Oka T, Takimoto E, Hayashi D, Hosoda T, Habara-Ohkubo A, Nakaoka T, et al: Bone morphogenetic proteins induce cardiomyocyte differentiation through the mitogen-activated protein kinase kinase kinase TAK1 and cardiac transcription factors Csx/Nkx-2.5 and GATA-4. Mol Cell Biol 19: 7096-7105, 1999.

13. Naito AT, Akazawa H, Takano H, Minamino T, Nagai T, Aburatani $\mathrm{H}$ and Komuro I: Phosphatidylinositol 3-kinase-Akt pathway plays a critical role in early cardiomyogenesis by regulating canonical Wnt signaling. Circ Res 97: 144-151, 2005.

14. Peng CF, Wei Y, Levsky JM, McDonald TV, Childs G and Kitsis RN: Microarray analysis of global changes in gene expression during cardiac myocyte differentiation. Physiol Genomics 9: 145-155, 2002

15. Alexander J and Stainier DY: A molecular pathway leading to endoderm formation in zebrafish. Curr Biol 9: 1147-1157, 1999.

16. Sinner D, Rankin S, Lee M and Zorn AM: Sox17 and beta-catenin cooperate to regulate the transcription of endodermal genes. Development 131: 3069-3080, 2004.

17. Yasunaga M, Tada S, Torikai-Nishikawa S, Nakano Y, Okada M, Jakt LM, Nishikawa S, Chiba T, Era T and Nishikawa S: Induction and monitoring of definitive and visceral endoderm differentiation of mouse ES cells. Nat Biotechnol 23: 1542-1550, 2005.

18. Kitajima S, Takagi A, Inoue T and Saga Y: MesP1 and MesP2 are essential for the development of cardiac mesoderm. Development 127: 3215-3226, 2000.

19. Semënov MV, Tamai K, Brott BK, Kühl M, Sokol S and He X: Head inducer Dickkopf-1 is a ligand for Wnt coreceptor LRP6. Curr Biol 11: 951-961, 2001. 
20. Sato N, Meijer L, Skaltsounis L, Greengard P and Brivanlou AH: Maintenance of pluripotency in human and mouse embryonic stem cells through activation of Wnt signaling by a pharmacological GSK-3-specific inhibitor. Nat Med 10: 55-63, 2004.

21. von Both I, Silvestri C, Erdemir T, Lickert H, Walls JR, Henkelman RM, Rossant J, Harvey RP, Attisano L and Wrana JL: Foxh1 is essential for development of the anterior heart field. Dev Cell 7: 331-345, 2004

22. Ng ES, Azzola L, Sourris K, Robb L, Stanley EG and Elefanty AG: The primitive streak gene Mixll is required for efficient haematopoiesis and BMP4-induced ventral mesoderm patterning in differentiating ES cells. Development 132: 873-884, 2005.

23. Okumura N, Akutsu H, Sugawara T, Miura T, Takezawa Y Hosoda A, Yoshida K, Ichida JK, Yamada M, Hamatani T, et al: $\beta$-catenin functions pleiotropically in differentiation and tumorigenesis in mouse embryo-derived stem cells. PLoS One 8: e63265, 2013.

24. Gubbay J, Collignon J, Koopman P, Capel B, Economou A, Münsterberg A, Vivian N, Goodfellow P and Lovell-Badge R: A gene mapping to the sex-determining region of the mouse $\mathrm{Y}$ chromosome is a member of a novel family of embryonically expressed genes. Nature 346: 245-250, 1990.
25. Marson A, Foreman R, Chevalier B, Bilodeau S, Kahn M, Young RA and Jaenisch R: Wnt signaling promotes reprogramming of somatic cells to pluripotency. Cell Stem Cell 3: $132-135,2008$.

26. Lluis F, Pedone E, Pepe S and Cosma MP: Periodic activation of Wnt/beta-catenin signaling enhances somatic cell reprogramming mediated by cell fusion. Cell Stem Cell 3: 493-507, 2008.

27. Bone HK, Nelson AS, Goldring CE, Tosh D and Welham MJ: A novel chemically directed route for the generation of definitive endoderm from human embryonic stem cells based on inhibition of GSK-3. J Cell Sci 124: 1992-2000, 2011.

28. Nakanishi M, Kurisaki A, Hayashi Y, Warashina M, Ishiura S, Kusuda-Furue $M$ and Asashima M: Directed induction of anterior and posterior primitive streak by Wnt from embryonic stem cells cultured in a chemically defined serum-free medium. FASEB J 23: 114-122, 2009.

29. Lin SY, Xia W, Wang JC, Kwong KY, Spohn B, Wen Y, Pestell RG and Hung MC: Beta-catenin, a novel prognostic marker for breast cancer: Its roles in cyclin D1 expression and cancer progression. Proc Natl Acad Sci USA 97: 4262-4266, 2000 . 